\title{
Research Paper: Psychological, Neurophysiological, and Mental Factors Associated With Gamma-Enhancing Neurofeedback Success
}

\author{
Zeynab Khodakarami $^{1^{*}}$ (D), Mohammad Firoozabadi² ${ }^{2}$ (C)
}

1. Department of Biomedical Engineering, Science and Research Branch, Islamic Azad University, Tehran, Iran

2. Department of Medical Physics, Faculty of Medical Sciences, Tarbiat Modares University, Tehran, Iran

\begin{tabular}{l|l}
$\begin{array}{l}\text { use vour device to scan } \\
\text { and read the article online }\end{array}$ & $\begin{array}{l}\text { Cittation: Khodakarami, Z., \& Firoozabadi, M. (2020). Psychological, Neurophysiological, and Mental Factors Associated } \\
\text { With Gamma-Enhancing Neurofeedback Success. Basic and Clinical Neuroscience, 11(5), 701-714. http://dx.doi.org/10.32598/ } \\
\text { bcn.11.5.1878.1 }\end{array}$ \\
dol'http://dx.doi.org/10.32598/bcn.11.5.1878.1
\end{tabular}

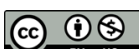

Article info:

Received: 27 May 2019

First Revision: 10 Jun 2019

Accepted: 02 Oct 2020

Available Online: 01 Sep 2020

Keywords:

Neurofeedback, BrainComputer Interface (BCI)illiteracy, Performance prediction, Fluid intelligence, Mental strategies

\begin{abstract}
$\underline{\text { A B S T RA C T }}$
Introduction: Regarding the neurofeedback training process, previous studies indicate that $10 \%-50 \%$ of subjects cannot gain control over their brain activity even after repeated training sessions. This study is conducted to overcome this problem by investigating inter-individual differences in neurofeedback learning to propose some predictors for the trainability of subjects.

Methods: Eight healthy female students took part in 8 (electroencephalography) EEG neurofeedback training sessions for enhancing EEG gamma power at the $\mathrm{Oz}$ channel. We studied participants' preexisting fluid intelligence and EEG frequency sub-bands' power during 2-min eyes-closed rest and a cognitive task as psychological and neurophysiological factors, concerning neurofeedback learning performance. We also assessed the self-reports of participants about mental strategies used by them during neurofeedback to identify the most effective successful strategies.
\end{abstract}

Results: The results revealed that a significant percentage of individuals ( $25 \%$ in this study) cannot learn how to control their brain gamma activity using neurofeedback. Our findings suggest that fluid intelligence, gamma power during a cognitive task, and alpha power at rest can predict gamma-enhancing neurofeedback performance of individuals. Based on our study, neurofeedback learning is a form of implicit learning. We also found that learning without a user's mental efforts to find out successful mental strategies, in other words, unconscious learning, lead to more success in gamma-enhancing neurofeedback.

Conclusion: Our results may improve gamma neurofeedback efficacy for further clinical usage and studies by giving insight about both non-trainable individuals and effective mental strategies.

\section{* Corresponding Author:}

Zeynab Khodakarami, MSc.

Address: Department of Biomedical Engineering, Science and Research Branch, Islamic Azad University, Tehran, Iran.

Tel: +98 (912) 5072015

E-mail: z.khodakarami@gmail.com 


\section{Highlights}

- A comprehensive literature review on the relationship between personal factors and neurofeedback success.

- We introduced some predictors for Gamma-enhancing neurofeedback performance of individuals.

- We proposed effective instructions for Gamma-enhancing neurofeedback learning.

\section{Plain Language Summary}

Using neurofeedback technique, people can learn to modulate their brain neural activity voluntarily, through a realtime feedback of their EEG and a reward to further motivate them. Neurofeedback has been proven to be efficient both in treatment of many different mental disorders and in cognitive enhancement of healthy participants. After almost six decades of neurofeedback research, some serious challenges in this area, such as neurofeedback-illiteracy or highly dependence of neurofeedback on user's individual characteristics, still remain unsolved and controversial. It has been shown that a non-negligible proportion of participants (estimated $10 \%-50 \%$ ) fail to gain significant control over their brain function through neurofeedback, even after several training sessions, which leads to useless and frustrating expenses. In this study, we introduced some predictors for estimating the success of individuals in learning neurofeedback before starting this procedure, as well as some guidelines for use in training procedure.

\section{Introduction}

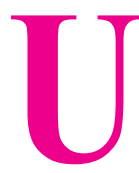

sing the neurofeedback technique, people can learn to modulate voluntarily their brain neural activity, through realtime feedback of their Electroencephalography (EEG) in the form of visual, auditory, or tactile information, and a reward during desirable changes in it. Using neurofeedback, different EEG frequency sub-bands activities could be modified (Berner, Schabus, Wienerroither, \& Klimesch, 2006; Keizer, Verment, \& Hommel, 2010; Leins et al., 2007; Raymond, Varney, Parkinson, \& Gruzelier, 2005; van Boxtel et al., 2012; Vernon et al., 2003). Neurofeedback has been proven to be efficient in the therapy of many different mental disorders such as attention-deficit hyperactivity disorder (Gevensleben et al., 2009), schizophrenia (Gruzelier, 2000), and anxiety (Moore, 2000). Also improved artistic (Egner \& Gruzelier, 2003; Raymond, Sajid, Parkinson, \& Gruzelier, 2005), sport-related (Arns, Kleinnijenhuis, Fallahpour, \& Breteler, 2008; Landers et al., 1991), and cognitive (Angelakis et al., 2007; Egner \& Gruzelier, 2004) functioning due to neurofeedback has been demonstrated in healthy participants.

Despite all advantages of neurofeedback, it has a main drawback. Gaining considerable long-lasting control on brain activity through neurofeedback requires a high number of training sessions, leading to high temporal, financial, and staff costs. Besides, previous studies re- vealed that a non-negligible proportion of participants (estimated 10\%-50\%) fail to gain significant control over their brain function through neurofeedback, even after several training sessions (Blankertz et al., 2010; Drechsler et al., 2007; Hammer et al., 2012; Hanslmayr, Sauseng, Doppelmayr, Schabus, \& Klimesch, 2005; Huster, Mokom, Enriquez-Geppert, \& Herrmann, 2013; Kober, Witte, Ninaus, Neuper, \& Wood, 2013; Lubar, Swartwood, Swartwood, \& O’Donnell, 1995; Neumann \& Birbaumer, 2003; Weber, Köberl, Frank, \& Doppelmayr, 2011). This phenomenon, first seen in Brain-Computer Interface (BCI) research, is called BCI-illiteracy (Blankertz et al., 2010; Kober et al., 2013; Witte, Kober, Ninaus, Neuper, \& Wood, 2013) or BCI-inefficiency (Hammer et al., 2012; Kübler, Blankertz, Müller, \& Neuper, 2011) in the literature, and has been demonstrated for both healthy and unhealthy participants, as for all EEG training features. In this context, the participants with and without the ability to learn self-regulation have been named as responders/non-responders, performers/ non-performers, or low/high aptitude users, respectively. Solving the BCI-illiteracy problem to avoid frustrating and costly training procedures is one of the biggest challenges in neurofeedback studies (Blankertz et al., 2010).

To solve the BCI-illiteracy problem, some research studies have been conducted in recent years to investigate whether there is any relationship between interindividual differences and the ability of people to learn neurofeedback self-regulation. Such studies aimed to propose some predictors for neurofeedback trainability. 
Table1. Summary of studies indicating the significant relationship between personal factors and neurofeedback performance

\begin{tabular}{|c|c|c|c|c|}
\hline & Personal Factor & EEG Trained Sub-Band & Study Population & Study \\
\hline \multirow{5}{*}{ 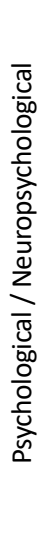 } & Measures of attention and memory span & Slow cortical potentials & Epilepsy patients & Daum et al. (1993) \\
\hline & Locus of control with regard to technology & Sensorimotor rhythm & Healthy subjects & Burde \& Blankertz (2006) \\
\hline & Intelligence quotient & Slow cortical potentials & $\begin{array}{l}\text { Amyotrophic lateral } \\
\text { sclerosis patients }\end{array}$ & Drechsler et al. (2007) \\
\hline & Motivational factors & $\begin{array}{l}\text { P300 / Sensorimotor } \\
\text { rhythm }\end{array}$ & $\begin{array}{l}\text { Amyotrophic lateral } \\
\text { sclerosis patients }\end{array}$ & Nijboer et al. (2010) \\
\hline & Locus of control with regard to technology & Sensorimotor rhythm & Healthy subjects & Witte et al. (2013) \\
\hline \multirow{3}{*}{ 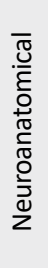 } & $\begin{array}{l}\text { Gray and white morphology of the } \\
\text { midcingulate cortex }\end{array}$ & Theta & Healthy subjects & Enriquez-Geppert et al. (2013) \\
\hline & $\begin{array}{l}\text { Structural integrity and myelination qual- } \\
\text { ity of deep white matter structures }\end{array}$ & Sensorimotor rhythm & Healthy subjects & Halder et al. (2013) \\
\hline & Gray and white matter volumes & $\begin{array}{l}\text { Sensorimotor rhythm/ } \\
\text { Gamma }\end{array}$ & Healthy subjects & Ninaus et al. (2015) \\
\hline \multirow{3}{*}{ 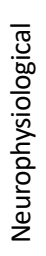 } & $\begin{array}{l}\text { Sensorimotor rhythm amplitude under } \\
\text { "relax with eyes open" }\end{array}$ & Sensorimotor rhythm & Healthy subjects & Blankertz et al. (2010) \\
\hline & $\begin{array}{l}\text { Low beta amplitude at eyes-open rest } \\
\text { / Beta-1 amplitude in the first 4.5-min } \\
\text { training block }\end{array}$ & Beta/Theta ratio & Healthy subjects & Nan et al. (2015) \\
\hline & Alpha amplitude at rest & Alpha & Healthy subjects & Wan et al. (2014) \\
\hline \multirow{3}{*}{ 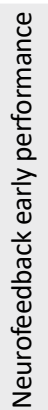 } & $\begin{array}{l}\text { Initial performance in Slow cortical } \\
\text { potentials self-regulation }\end{array}$ & Slow cortical potentials & $\begin{array}{l}\text { Amyotrophic lateral } \\
\text { sclerosis patients }\end{array}$ & Neumann \& Birbaumer (2003) \\
\hline & $\begin{array}{l}\text { Slow cortical potentials self-control in } \\
\text { the training session } 3\end{array}$ & Slow cortical potentials & $\begin{array}{l}\text { Amyotrophic lateral } \\
\text { sclerosis patients / } \\
\text { healthy subjects }\end{array}$ & Kübler et al. (2004) \\
\hline & $\begin{array}{c}\text { Sensorimotor rhythm self-control in the } \\
\text { training session } 9\end{array}$ & Sensorimotor rhythm & Healthy subjects & Weber et al. (2011) \\
\hline
\end{tabular}

Finding such factors that may serve to predict neurofeedback performance, on the one hand, results in a better understanding of both BCI-illiteracy phenomenon and underlying neurophysiological and behavioral mechanisms involved in brain self-regulation (Blankertz et al., 2010; Neumann \& Birbaumer, 2003), and on the other hand, until the problem of BCI-illiteracy is solved, utilizing neurofeedback alternatives for participants with less probability of success, may serve to avoid the costly and frustrating procedures. Previous studies have introduced predictors for neurofeedback learning perfor- mance based on four following factors (a summary can be found in Table 1):

1. Psychological/neuropsychological traits (Burde \& Blankertz, 2006; Daum et al., 1993; Drechsler et al., 2007; Hammer et al., 2012; Nijboer, Birbaumer, \& Kübler, 2010; Witte et al., 2013).

2. Brain structural and neuroanatomical factors (Enriquez-Geppert et al., 2013; Halder et al., 2013; Ninaus et al., 2015); 
3. Brain neurophysiological parameters in the form of brain activity (Blankertz et al., 2010; Nan, Wan, Vai, \& Da Rosa, 2015; Wan, Nan, Vai, \& Rosa, 2014);

4. Neurofeedback early training performance (Kübler, Neumann, Wilhelm, Hinterberger, \& Birbaumer, 2004; Neumann \& Birbaumer, 2003; Weber et al., 2011).

Mental strategies used by an individual during a neurofeedback session to gain self-control is another core feature of successful training. A proper mental strategy can facilitate and accelerate neurofeedback learning. In typical BCI applications, very specific instructions can be transmitted to the participants by the experimenters (Kober et al., 2013). But the mental strategies used in neurofeedback studies by participants and their effects are scarcely investigated (Kober et al., 2013; Nan et al., 2012). In the few studies conducted to assess the effectiveness of different mental strategies, positive strategies (e.g. positive thinking or positive emotions) and also no conscious and explicit mental strategy has been proven as successful ones (Angelakis et al., 2007; Birbaumer, Ruiz, \& Sitaram, 2013; Kober et al., 2013; Nan et al., 2012; Neumann \& Birbaumer, 2003; Rubik, 2011; Witte et al., 2013). Table 2 presents a summary of the studies that explored successful mental strategies in neurofeedback learning. As can be seen from both Table 1 and Table 2, most studies investigated the association between personal factors and neurofeedback performance have been focused on Sensorimotor Rhythms (SMR) and Slow Cortical Potentials (SCP) self-regulation, while personal factors associated with success in self-regulation of other EEG sub-band activities have seldom been studied.

The ability of individuals to enhance EEG gamma power using neurofeedback and subsequently improvement of focused arousal, memory, visual feature binding, and fluid intelligence have been reported before (Bird, Newton, Sheer, \& Ford, 1978; Elliott \& Müller, 1998; Keizer, et al., 2010; Keizer, Verschoor, Verment, \& Hommel, 2010; Khodakarami \& Firoozabadi, 2014; Rubik, 2011; Sams, 1995; Staufenbiel, Brouwer, Keizer, \& van Wouwe, 2014; Tallon-Baudry, Bertrand, Peronnet, \& Pernier, 1998). Visual feature binding phenomenon plays a key role in human visual perception (Hommel, 1998). Gamma neurofeedback illiteracy and possible effects of personal factors on it have rarely been investigated. This study aimed to investigate the relationship between inter-individual differences and gamma neurofeedback trainability of subjects. To do this, we examined the association between some psychological, neurophysiological, and mental factors and neurofeedback success in both trainable and non-trainable individuals, to define some reliable predictors for gamma-enhancing neurofeedback performance.

\section{Materials and Methods}

\subsection{Study participants}

Eight healthy female students voluntarily participated in this study (Mean \pm SD age: $24.46 \pm 2.35$ years; 2 lefthanded). All participants were neurofeedback- and BCInovices and had a normal or corrected-to-normal vision, with no history of neurological diseases (e.g. migraine or epilepsy) and no medication use that could influence cognitive performance or brain activity during the study. Before the experiments, all participants were informed about the purpose of the study and gave written informed consent to the study protocol. This study conforms with the World Medical Association Declaration of Helsinki.

\subsection{Study procedure}

All subjects participated in a pre-test neurophysiological and psychological factors assessment session. In that session, a 2-min baseline EEG was recorded during both eyes-closed rest and a cognitive task (pre-test baseline). The cognitive task was based on mental search and counting upward blue numbers among red numbers represented on a 17-inch computer screen, one meter away from the subject. After the baseline EEG recordings, the subject's fluid intelligence was measured by using Cattell's Culture-Fair Intelligence Test (Cattell, Krug, $\&$ Barton, 1973). At the beginning of the session before performing the intelligence test, the subjects filled out a questionnaire asked about their last night's sleep, health issues, and whether they are mentally prepared for participating in a psychological test (pre-test questionnaire). The pre-test sessions were conducted at 9-11 AM, at the neurofeedback lab of the Biomedical Engineering Department of the Science and Research Branch of Islamic Azad University.

After the end of all pre-test sessions, each subject participated in 8 gamma-enhancing neurofeedback sessions. At each session, before the start of neurofeedback training, a 2-min baseline EEG at eyes-closed rest was recorded from the subject (pretraining baseline), identical to the pre-test EEG recordings. Subjects were also asked to fill out two pretraining and posttraining questionnaires at the beginning and the end of each session, respectively. In the pretraining questionnaire, any notable changes possibly due to neurofeedback training in appetite, sleep pattern, memory capacity, and mental and cognitive ability during previous days were questioned. The posttraining questionnaire enquired for any mental strategies the subject applied for gaining control over the feedback during the session, and the success of them on a 
Table 2. Summary of studies suggesting effective mental strategies for neurofeedback success

\begin{tabular}{|c|c|c|c|}
\hline \multirow{2}{*}{ Successful Strategies } & EEG Trained Sub-band & Study Population & \multirow{2}{*}{ Study } \\
\hline & \multicolumn{2}{|c|}{ Mental Strategy (Personal Factor) } & \\
\hline $\begin{array}{l}\text { Neither instructions nor explicit mental } \\
\text { strategies }\end{array}$ & $\begin{array}{l}\text { Review on real-time functional mag- } \\
\text { netic resonance imaging neurofeed- } \\
\text { back of different bands }\end{array}$ & $\begin{array}{l}\text { Healthy subjects / } \\
\text { Animals }\end{array}$ & Birbaumer et al. (2013) \\
\hline No conscious mental strategy & Slow cortical potentials & $\begin{array}{l}\text { Amyotrophic lateral } \\
\text { sclerosis patients }\end{array}$ & $\begin{array}{c}\text { Neumann \& Birbaumer } \\
(2003)\end{array}$ \\
\hline No conscious mental strategy & Sensorimotor rhythm & Healthy subjects & Witte et al. (2013) \\
\hline $\begin{array}{l}\text { Positive strategies (positive thoughts) } \\
\text { and no strategy }\end{array}$ & Alpha & Healthy subjects & Angelakis et al. (2007) \\
\hline Positive thinking & Alpha & Healthy subjects & Nan et al. (2012) \\
\hline Positive emotions (e.g. happiness) & Gamma & Healthy subjects & Rubik (2011) \\
\hline No mental strategy & Sensorimotor rhythm & Healthy subjects & Kober et al. (2013) \\
\hline
\end{tabular}

5-point Likert-type scale (0: No control, 5: Full control). In posttraining questionnaires, the subjects again rated their motivation for gaining control over the feedback, on a 5-point Likert-type scale ( 0 : Not at all motivated, 5: Extremely motivated). An overview of the experimental design of the current study can be found in Figure 1.

\subsection{Intelligence assessment}

Fluid intelligence was tested by using the paper-based version of Cattell's Culture-Fair Intelligence Test (Scale 3) Form A (Cattell et al., 1973). This is a non-verbal and culture-independent test, with 50 multiple choice questions and 14-min test time. The test is composed of four individually timed subtests of series, classification, matrices, and conditions, with questions of increasing difficulty. The results of the test were calculated in terms of accuracy for each subject.

\subsection{Baseline EEG recordings}

Both pre-test and pretraining baseline EEG signals were recorded from the $\mathrm{Oz}$ electrode according to the international 10-20 system using BioGraph Infiniti

A)

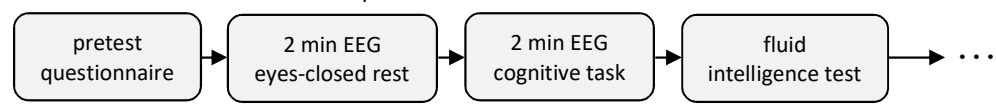

B)

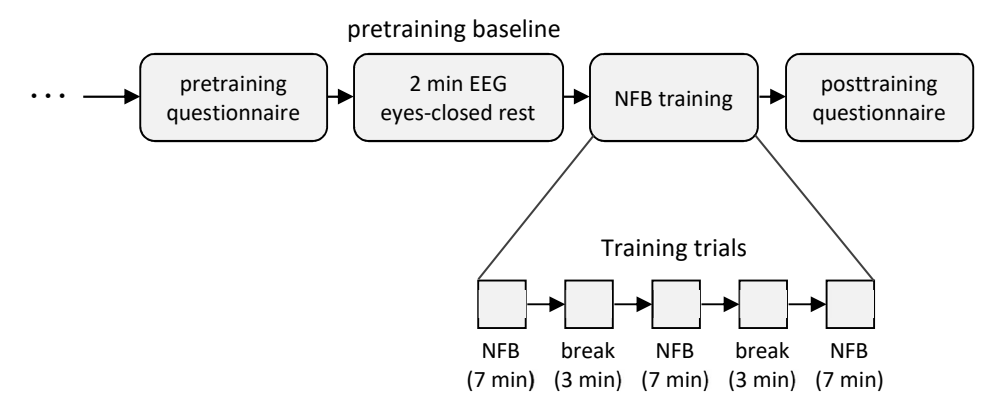

8 Gamma-enhancing neurofeedback sessions:

Figure 1. Schematic representation of the study experimental design

NEUR

A: The procedure of the pre-test session; B: The procedure of a single neurofeedback training session. Each subject participated in 8 gamma-enhancing neurofeedback sessions, three training sessions per week. 
Table 3. Descriptive statistics of study participants.

\begin{tabular}{|c|c|c|c|c|c|}
\hline \multirow{2}{*}{ Variable } & \multicolumn{2}{|c|}{ All $(n=8)$} & \multirow[t]{2}{*}{ Responders $(n=6)$} & \multirow[t]{2}{*}{ Non-Responders $(n=2)$} & \multirow{2}{*}{ P (Mann-Whitney) ${ }^{\mathrm{b}}$} \\
\hline & Mean士SD & $\mathrm{P}(\mathrm{K}-\mathrm{S})^{\mathrm{a}}$ & & & \\
\hline Age, y & $24.46 \pm 2.35$ & 0.20 & $24.05 \pm 2.61$ & $25.69 \pm 0.74$ & 0.50 \\
\hline $\mathrm{BMI}, \mathrm{kg} / \mathrm{m}^{2}$ & $20.65 \pm 2.62$ & 0.20 & $19.46 \pm 1.47$ & $24.19 \pm 1.93$ & $0.04^{*}$ \\
\hline IQ & $103.50 \pm 22.58$ & 0.20 & $112.17 \pm 18.33$ & $77.50 \pm 9.19$ & $0.04^{*}$ \\
\hline Gamma changec & - & - & $2.24 \pm 1.52$ & $-0.29 \pm 0.07$ & $0.04 *$ \\
\hline
\end{tabular}

${ }^{a}$ Results from the K-S test assessing the normal distribution of variables;

NEURCIENCE

${ }^{b}$ Results from the Mann-Whitney U test examining significant differences between responders and non-responders groups;

${ }^{c}$ Mean gamma power change from pretraining to training over 8 neurofeedback sessions $\left(\mu \mathrm{V}^{2}\right)$;

${ }^{*} \mathrm{P}<0.05$.

(Thought Technology Ltd, Montreal, QC) and golden electrodes, sampled at $256 \mathrm{~Hz}$. The ground electrode was located at the right earlobe, and the reference was at the left earlobe. Electrode impedances were kept below $5 \mathrm{k} \Omega$. During EEG recordings, the subjects were sitting still in a comfortable chair with arms lying relaxed on armrests.

\subsection{Neurofeedback training}

Eight gamma-enhancing neurofeedback sessions were conducted for each subject, three training sessions per week. Each training session consisted of three 7-min trials, with 3-min breaks between. In each trial, the EEG signal recorded from the $\mathrm{Oz}$ electrode (according to the international 10-20 system) was filtered to gamma (36$44 \mathrm{~Hz}$ ) and beta $(12-20 \mathrm{~Hz})$ frequency bands using two elliptic filters. Then, gamma and beta signal peak-to-peak amplitudes fed real-time back to the subject, with the aim of gamma enhancing and beta suppression simultaneously. We defined gamma frequency band around 40 $\mathrm{Hz}$ because it is the most widely accepted indicator of the gamma range (Başar-Eroglu, Strüber, Schürmann, Stadler, \& Başar, 1996; Hammond, 2000; Sams, 1995; Tallon-Baudry \& Bertrand, 1999). It has been suggested in previous studies that the beta frequency band power will be changed because of the gamma frequency band power changes (Bird et al., 1978; Keizer, et al., 2010). Therefore, we included beta suppression in our gammaenhancing neurofeedback protocol, to investigate just gamma-band activity self-regulation. The width of the gamma and beta bands were made identical, to prevent possible effects of a bandwidth difference in the gamma and beta band (Keizer et al., 2010).
The neurofeedback paradigm was generated by using the BioGraph Infiniti Multimedia Biofeedback Software (Thought Technology Ltd, Montreal, QC). During the neurofeedback training procedure, two thresholds were implemented, one applied on the gamma and the other on the beta signal peak-to-peak amplitude. The gamma and beta threshold was set automatically to the value that the gamma and beta signal peak-to-peak amplitude surpassed above and below it respectively, in $60 \%$ of the time during the preceding 30 seconds window. Whenever the gamma peak-to-peak amplitude exceeded above the gamma threshold and at the same time, the beta peak-topeak amplitude exceeded below the beta threshold, for at least $250 \mathrm{~ms}$, the subject received some visual and auditory rewards represented on a 17-inch computer screen 1 meter away from her. The subjects were only instructed to be mentally focused and physically relaxed during neurofeedback training. They could utilize any mental strategy they wanted to gain as more rewards as possible.

During neurofeedback training, two online filters over the frequency ranges of $36-44 \mathrm{~Hz}$ and $12-20 \mathrm{~Hz}$ were applied to the recorded Oz-EEG signal to extract gamma and beta activity, respectively. Movement artifacts caused by muscle contraction (EMG) is a significant source of noise at these frequency ranges, especially over the gamma band, which can cause a false-positive error in neurofeedback training. To ensure that gamma and beta activity is properly extracted and used in neurofeedback training, we first asked all subjects to sit completely relaxed in a comfortable chair throughout the neurofeedback experiments, with no stress or extra muscle contraction or movement. We also carefully monitored the behavior of all subjects during neurofeedback training, to exclude signal intervals identified as being contaminated 
with artifacts, for further analysis. The second approach we used to prevent augmentation of the gamma or beta signal by muscle artifacts was to apply an online artifact rejection threshold, which is a nonlinear filter, on the EEG signal during training. The artifact rejection threshold was identified for each subject individually, at the beginning of each training session. For this reason, the subjects were asked to perform tasks such as blinking, or contraction of the facial muscles, while the experimenter set the artifact threshold to the value on the 50-64 Hz filtered EEG signal peak-to-peak amplitude that the amplitude surpassed above it during the contraction tasks. The subjects were not rewarded when the amplitude exceeded above the defined artifact threshold during the training, even if the gamma and the beta amplitudes satisfied the neurofeedback training goal. Other techniques, such as adaptive filters, can also be used to remove artifacts from EEG signals, which are more computationally expensive and was not adopted in this study.

\subsection{Data analysis}

The subjects were physically relaxed and still during the EEG signals recording. Therefore, only a $0.2-48 \mathrm{~Hz}$ band-pass Butterworth filter and then visual inspection was used for artifact rejection. The absolute power of the theta $(4-8 \mathrm{~Hz})$, alpha $(8-12 \mathrm{~Hz})$, beta $(12-20 \mathrm{~Hz})$, and gamma $(36-48 \mathrm{~Hz})$ frequency bands were extracted from pre-test baseline EEG signals to be investigated as neurophysiological predictors. We also explored any possible association between participants' neurofeedback performance and both fluid intelligence and mental strategies used by them during training. To investigate the effects of neurofeedback training on subjects' brain activity, gamma absolute power of pretraining baseline, and during training EEG signals were extracted. EEG signals' frequency bands' absolute power was estimated using the Welch algorithm and MATLAB R2013a software (MathWorks, Inc., Natick, MA, USA).

To identify responders and non-responders to neurofeedback training, the mean gamma absolute power change from pretraining to the training of each session was calculated for each subject. A subject was assigned to the responders' group if the mean gamma power change was positive for both over 8 sessions and each session separately, which means that gamma power during each training session was controlled and increased. Five subjects reached the criterion and categorized as responders. In contrast, a subject was assigned to the non-responders group if the mean gamma power change over 8 sessions was negative. Two subjects showing a decrease in gamma power over 5 sessions of 8 , were categorized as non-responders. The mean gamma power change of the last subject was positive over 8 sessions but negative for 2 sessions of the total 8 sessions. Considering that one of these sessions was the last training one, we categorized this subject as the poor responder.

In this study, we employed 4 types of statistical analysis, using IBM SPSS V. 21 (SPSS, Inc., Chicago, IL, USA): 1. The Kolmogorov-Smirnov (K-S) test to check for normal distribution of the variables; 2 . The paired samples $t$ test, Wilcoxon and Mann-Whitney $U$ tests to examine significant differences; 3. The Pearson correlation analysis to evaluate significant statistical relationships; 4. The simple linear and quadratic regression analysis to identify statistical trends.

\section{Results}

\subsection{Neurofeedback Performance: Responders vs. Non-responders}

Subjects' age and Body Mass Index (BMI) were both normally distributed conformed by the K-S result (age: $\mathrm{z}=0.430$; BMI: $\mathrm{z}=0.518 ; \mathrm{P}>0.05)$, with the mean of $24.46 \pm 2.35$ years and $20.65 \pm 2.62 \mathrm{~kg} / \mathrm{m}^{2}$, respectively (Table 3). Based on the Mann-Whitney $U$ test, there was no significant difference between the age of the two groups of responders and non-responders, while a significant difference was found in BMI between the two groups (age: $\mathrm{z}=-0.67 ; \mathrm{P}>0.05$; BMI: $\mathrm{z}=-2.00 ; \mathrm{P}<0.05$ ) (Table 3). Based on pre-test and pretraining questionnaires, there were no side effects of neurofeedback training on subjects and all were physically and mentally prepared for participating in the experiments. Examining posttraining questionnaires revealed the very motivated and extremely motivated levels for all subjects in all sessions.

Using the paired t test, a significant increase in gamma power from pretraining to training across 8 sessions was found for each responder $(\mathrm{P}<0.01)$, in the absence of a significant difference for each non-responder $(\mathrm{P}>0.05)$. The poor responder subject showed an increase in gamma power over 8 sessions at the significance level of $\mathrm{P}=0.075$, suggesting that she could not control her gamma activity completely. The Wilcoxon test revealed a significant difference between mean gamma power of pretraining and training across 8 sessions for responders (including the poor responder) $(\mathrm{z}=-2.20 ; \mathrm{P}<0.05)$, while no significant difference for non-responders was achieved $(\mathrm{z}=-1.34 ; \mathrm{P}>0.05)$ (Figure $2 \&$ Table 3$)$. 


\section{Responders vs. Non-responders Performance}

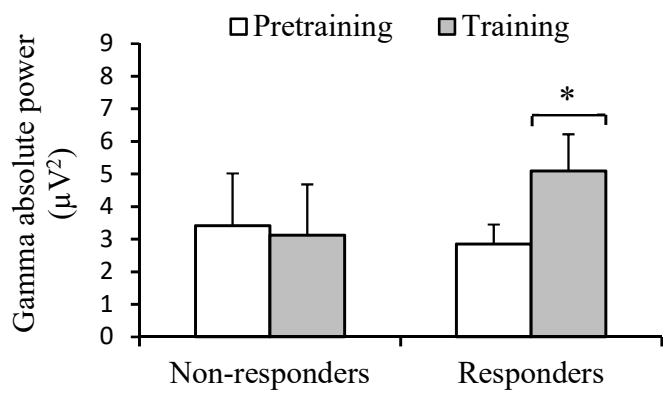

NEUR SCIENCE

Figure 2. Mean gamma absolute power over 8 sessions. Unlike non-responders, responders could significantly increase their gamma activity over training sessions. Error bars represent the standard error of the mean. The asterisk indicates a significance level of $\mathrm{P}<0.05$.

\subsection{Psychological factor: Fluid intelligence}

The subjects' Intelligence Quotient (IQ) reflecting the fluid intelligence was normally distributed confirmed by the K-S $(\mathrm{z}=0.512 ; \mathrm{P}>0.05)$, with the mean value of $103.50 \pm 22.58$ (Table 3). Using the Mann-Whitney U test, a significant difference in IQ was found between the responders and non-responders groups $(\mathrm{z}=-2.01 ; \mathrm{P}<0.05)$ (Table 3). As seen in Figure 3A, responders have a significantly higher IQ than non-responders (responders' mean percentage correct: $51.33 \pm 8.64$; non-responders' mean percentage correct: $33.00 \pm 7.07$ ). The Pearson test revealed a significant positive correlation between subjects' pre-existing fluid intelligence and mean gamma power change from pretraining to training over 8 neurofeedback sessions $(\mathrm{r}=0.75 ; \mathrm{n}=8 ; \mathrm{P}<0.05)$ (Figure $3 \mathrm{~B}$ ). This effect reflects more gamma power increase and more success in neurofeedback for people with higher IQ.
The results indicate that fluid intelligence has predictive value for estimating gamma neurofeedback performance of people, and can be used for classifying subjects as responders or non-responders before training. Based on the mathematical relationship between intelligence and gamma power change, the user's mean gamma power increase during neurofeedback sessions can be predicted. However, based on the Pearson analysis results, the fluid intelligence factor is only accounted for $56 \%$ of the variance $\left(\mathrm{R}^{2}\right)$ in mean gamma power increase suggesting that other influencing factors contribute to self-regulating brain gamma activity. Confirming this, the IQ of the poor responder subject was higher than the average IQ of all subjects, but she could not successfully make a significant increase in her brain gamma power over neurofeedback sessions.

\subsection{Neurophysiological factor: Brain neural ativity}

To identify potential neurophysiological predictors for gamma neurofeedback performance, we studied statistical relationships between gamma, beta, alpha, and theta absolute power extracted from pre-test EEG signals of two cognitive and relaxed conditions and mean gamma power change over 8 training sessions, using the Pearson correlation. The results showed a significant strong positive correlation between cognitive task gamma absolute power and mean gamma power change of a group of 6 subjects, excluding one non-responder and the poor responder subjects as outliers $(\mathrm{r}=0.88 ; \mathrm{n}=6 ; \mathrm{P}=0.02)$ (Figure 4A). Another significant correlation was found between at rest alpha absolute power and mean gamma power change of a group of 7 subjects, at a significance level of $\mathrm{P}=0.06$ ( $\mathrm{r}=-0.72 ; \mathrm{n}=7 ; \mathrm{P}=0.06$ ) (Figure 4B). No significant correlations were found between the mean gamma power change and any of the other frequency bands' powers $(\mathrm{P}>0.05)$.
A)

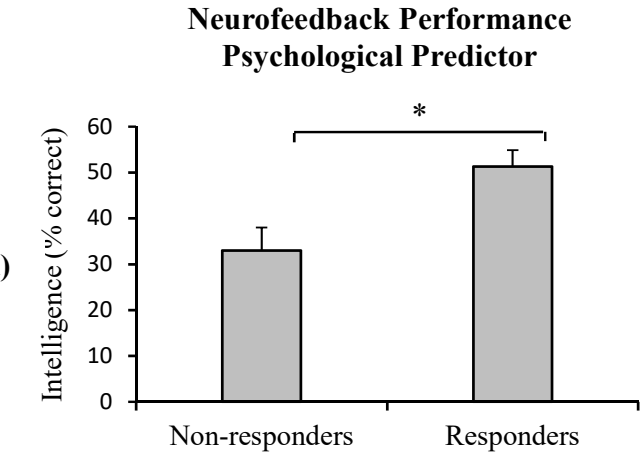

Neurofeedback Performance Psychological Predictor

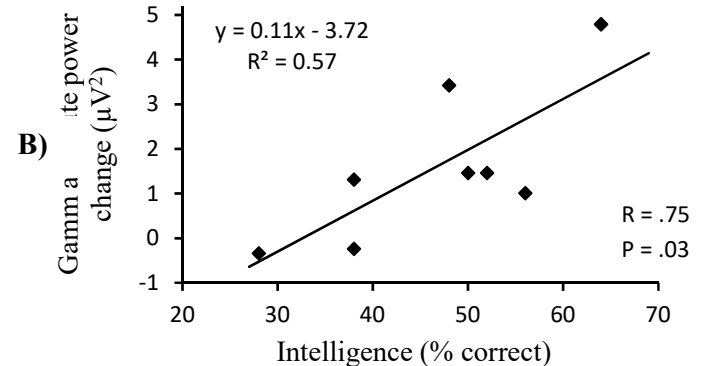

NEUR SCIENCE

Figure 3. Fluid intelligence effects on gamma-enhancing neurofeedback performance A: Responders' IQs are significantly higher than non-responders' $(\mathrm{P}<0.05)$; B: A significant positive correlation between fluid
intelligence and mean gamma power increase of all subjects over 8 neurofeedback sessions. The solid line results from a linear regression. 
Neurofeedback Performance Neurophysiological Predictor

A)

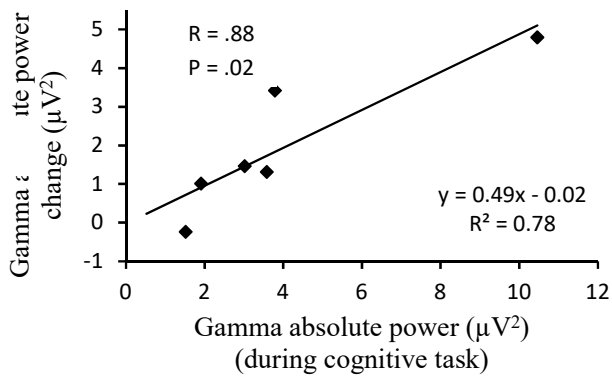

B)

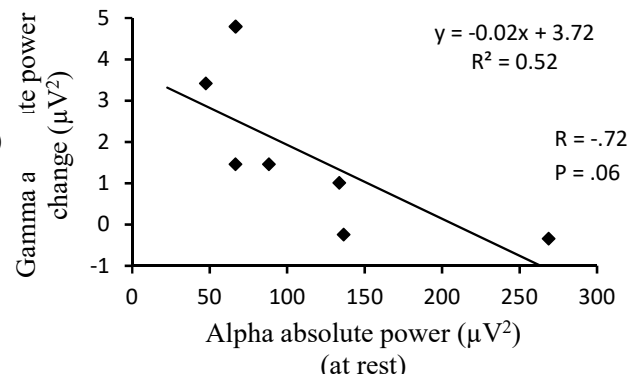

NEUR:SCIENCE

Figure 4. The statistical relationship between brain neuronal activity and gamma-enhancing neurofeedback performance

A: A significant positive correlation between cognitive task gamma absolute power and mean gamma power change of subjects over 8 neurofeedback sessions; B: A significant negative correlation between alpha absolute power during rest and gamma power change of subjects over 8 neurofeedback sessions. Solid lines result from a linear regression.

\subsection{Mental strategy: Conscious vs. Unconscious learning}

We studied posttraining questionnaires to investigate the effects of different mental strategies on gamma-enhancing neurofeedback performance. In self-reports, the subjects had written down the strategies they used during neurofeedback sessions to control their gamma activity and the effect of each strategy. Based on the subjects' descriptions, successful strategies were related to "focus on different parts of feedback image", "concentration on image variations", "predicting next feedback image", "rapid thinking and mental activity", and "no strategy". The following results have been extracted from self-reports:
- Different strategies were used by most subjects, especially in initial sessions. A distinct strategy necessarily did not have the same effects on the performance of different subjects. For example, one strategy might prove successful for one subject, but not for the rest.

- Using a successful strategy repeatedly did not lead to success again. Accordingly, after a while of using an effective strategy, one was forced to change the strategy to achieve favorable results.

- The intense mental effort resulted in less success.
Neurofeedback Conscious vs.

\section{Unconscious Learning}

A)

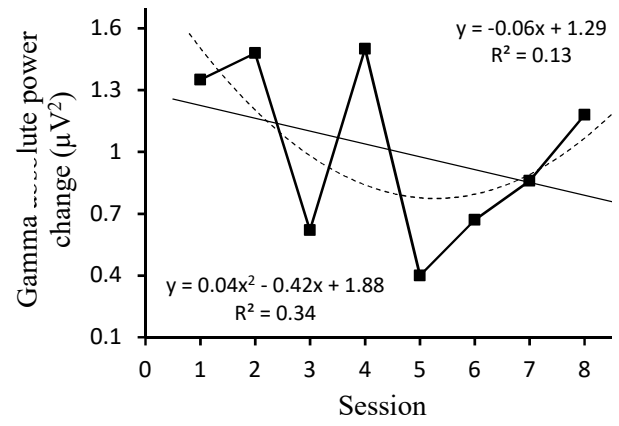

B)

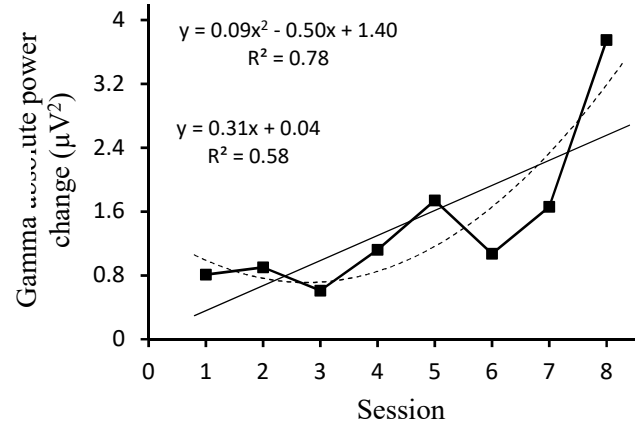

Figure 5. Gamma's absolute power change over 8 neurofeedback sessions

NEUR:SCIENCE

A: Conscious learning curve for one of the subjects using specific mental strategies during neurofeedback training. Neither linear nor quadratic trends are significant; B: Unconscious learning curve for the subject using no specific mental strategy during neurofeedback training. Both linear and quadratic trends are significant, while the quadratic trend line shows better fitting. Solid lines indicate a linear trend and dashed lines quadratic trend. 
- Most subjects could control their gamma activity without using any specific mental strategy in the final sessions. Subjects reported this condition as "I didn't do any specific mental task", "My mind was not involved in anything", and "I didn't think at all and just focused on purpose". Because of less mental effort and fatigue, they found this condition more comfortable and desirable.

- One subject did not use any specific strategy during any of the sessions. She often felt that she was not involved in the training process and could not establish the relationship between her mental states and feedback variations. The subject belonged to the responders' group and was the only one who learned neurofeedback unconsciously. Other subjects tried to learn neurofeedback consciously by trying to find relationships between their mental states and the feedback status.

To investigate gamma self-regulation conscious and unconscious learning in detail, individual linear and quadratic trends as an indicator of learning were calculated on the mean gamma power changes from pretraining to training across 8 sessions, utilizing regression analysis. Both linear $\left(\mathrm{F}_{1,6}=8.49 ; \mathrm{P}=0.03 ; \mathrm{R}^{2}=0.59\right)$ and quadratic $\left(\mathrm{F}_{2,5}=8.74 ; \mathrm{P}=0.02 ; \mathrm{R}^{2}=0.78\right)$ trends proved significant for the no strategy subject (unconscious learner), but not for any other subjects (conscious learners) $(\mathrm{P}>0.05$ ). Learning curves for one of the conscious learners and the unconscious learner are depicted in Figure 5A and B, respectively. The results suggest that using no strategy during gamma neurofeedback training results in better learning through a significant increase in gamma power from session to session. Higher $\mathrm{R}^{2}$ of the quadratic trend line $(0.78)$ compared to the linear trend line $(0.58)$ for the no strategy learning curve indicated better fitting for the quadratic model, suggesting an increase in gamma selfregulation learning rate in the final sessions.

\section{Discussion}

To solve the BCI-illiteracy problem in neurofeedback applications, previous studies proposed some psychological/ neuropsychological (Burde \& Blankertz, 2006; Daum et al., 1993; Hammer et al., 2012; Nijboer et al., 2010; Witte et al., 2013), neuroanatomical (Enriquez-Geppert et al., 2013; Halder et al., 2013), and neurophysiological (Blankertz et al., 2010) factors besides neurofeedback initial performance (Kübler et al., 2004; Neumann \& Birbaumer, 2003; Weber et al., 2011) as the predictor of individuals' neurofeedback trainability. The mental strategy used by an individual during a neurofeedback session is another factor that influences on gaining brain self-control (Angelakis et al., 2007; Birbaumer et al., 2013; Kober et al., 2013; Nan et al., 2012; Neumann \& Birbaumer, 2003; Rubik, 2011; Witte et al., 2013). The percentage of non-responders to neurofeedback training of different frequency bands have been reported as $10 \%-50 \%$, including healthy and unhealthy subjects (Blankertz et al., 2010; Drechsler et al., 2007; Hammer et al., 2012; Hanslmayr et al., 2005; Huster et al., 2013; Kober et al., 2013; Lubar et al., 1995; Neumann \& Birbaumer, 2003; Weber et al. 2011). Gamma neurofeedback illiteracy has rarely been investigated in studies. In the current study, $25 \%$ of participants were identified as non-responders to gamma neurofeedback. The proportion of non-responders identified in each study depends on the defined performance criterion. Because of the non-uniform increase in the gamma power over training sessions of participants in this study, we used the power increase in all sessions as the performance criterion. Based on this criterion, the two identified groups of responders and non-responders were statistically significantly different in mean gamma power increase over training sessions.

Our results indicated that individuals who have higher fluid intelligence could perform better in learning gamma self-control through neurofeedback. This finding confirms the role of fluid intelligence in identifying new relationships and skills acquisition (Valentin Kvist \& Gustafsson, 2008; Watkins, Lei, \& Canivez, 2007). In contrast to our results, Keizer et al. (2010) did not find any significant correlation between individuals' pre-test fluid intelligence and gamma power change through neurofeedback. They examined gamma power increase from pre-test to posttest at rest condition, but we instead focused on gamma self-regulation during neurofeedback, directly. Drechsler et al. (2007) reported no significant correlation between IQ measures and the selfregulation performance of participants. They employed a short form of the German WISC III as the IQ test, containing both fluid and crystallized intelligence measures that were not comparable with our study in which only fluid intelligence was tested.

Our findings also suggested that gamma power during cognitive task and alpha power at rest might be a predictor of gamma neurofeedback performance. Supporting our results, other studies have also reported a significant correlation between neurofeedback performance and brain neural activities measured before training (Blankertz et al., 2010; Nan et al., 2015; Wan et al., 2014). Assessing frequency bands' powers as the predictor of neurofeedback performance has some limitations. Brain potentials are affected by transient factors such as the current level of fatigue or attention, or mental states. Hence, it seems more reliable to estimate each neuro- 
feedback session performance based on brain potentials measured before the same session or more complex factors with less dependency on mental states.

Studying posttraining self-reports of participants about the effectiveness of strategies they used during neurofeedback training revealed that:

Visual strategies involved in image perception and memory restoration are possibly successful strategies for individual gamma-enhancing neurofeedback. This finding is in accordance with previous findings implicated that brain neural synchronization in the gamma band is associated with visual objects perception, visual working memory, and also short-term and long-term memory (Elliott \& Müller, 1998; Keizer, et al., 2010; Sams, 1995; TallonBaudry \& Bertrand, 1999; Tallon-Baudry et al., 1998).

Although participants in initial sessions have used different strategies, they found that conducting later sessions with no intense mental effort and specific strategy was more successful and comfortable. This finding is in line with the theory that neurofeedback learning can be viewed as skill learning (Birbaumer et al., 2013; Kober et al., 2013); brain self-regulation is originally based on trialand-error. Then, the learned self-regulation skill stores in the implicit memory and its retrieval requires no conscious and effortful search.

Our results showed that unconscious learners had better performance compared to other conscious learners. Moreover, both intense mental effort and repeating successful strategies by participants failed to gain appropriate brain self-control. Probably employing conscious mental strategies by participants, especially in later sessions, overload cognitive resources, which impedes complete automatization of self-regulation skill acquisition (Kober et al., 2013; Neumann \& Birbaumer, 2003). Accordingly, although the IQ of the poor responder participant was above the average IQ of all participants, her poor performance might be due to spending too much effort in using different types of strategies even in final sessions (mentioned by her in her self-report). Supporting this finding, the effectiveness of using no conscious mental strategy in neurofeedback learning of other frequency bands (e.g. SCP, SMR, and alpha) have also been demonstrated (Angelakis et al., 2007; Birbaumer et al., 2013; Kober et al., 2013; Neumann \& Birbaumer, 2003; Witte et al., 2013).

Another finding of this study was that a distinct strategy had different effects on the performance of different individuals. Nan et al. (2012) also mentioned this finding in their alpha neurofeedback study. Regarding this finding and the efficacy of unconscious neurofeedback learning, we support the conclusion of other studies (Birbaumer et al., 2013; Kober et al., 2013; Neumann \& Birbaumer, 2003; Witte et al., 2013) about providing neurofeedback participants with no explicit limiting instructions before training and just ask them to be physically relaxed while mentally focused without any hard mental efforts.

One advantage of this study compared to previous studies is that we assessed personal factors in relation to neurofeedback performance over 8 training sessions, while most previous studies just investigated neurofeedback performance in one training session. Skill learning occurred through time. Moreover, transient factors such as the current level of fatigue or attention influence both brain potentials and learning in one session. Therefore, assessing neurofeedback overall performance over several training sessions seems more reliable in such studies. Another advantage of the current study to other studies predicting neurofeedback later performance from initial performance is that the predictive factors introduced here for estimating neurofeedback trainability can be measured before the neurofeedback training course. In this way, we can avoid conducting any initial neurofeedback sessions with the risk of useless temporal and financial costs for non-responders. Second, the steadiness or variability of the participants' learning rate should be considered in studies focusing on the predictive value of neurofeedback early performance, which was rarely investigated before (Kübler et al., 2004). One important limitation of our study is the small number of participants. Some results have been reported on two non-responder subjects or only one unconscious learner. Another limitation was that our participants were only females. Hence, our findings should be considered preliminary and their utilization in clinical and experimental setups needs further validation.

\section{Conclusion}

In summary, in this study, we introduce three predictors for estimating gamma-enhancing neurofeedback trainability of individuals: 1. Fluid intelligence; 2. Gammaband power of EEG during a cognitive task; and 3. Alpha band power of EEG at eyes-closed rest. According to our results, a significant number of individuals cannot control their brain activity through gamma neurofeedback. The proposed psychological and neurophysiological predictors in this study can be used for classifying subjects as responders or non-responders before gamma neurofeedback training, avoiding to conduct any useless training sessions. Another important and applicable finding of this research is that employing no specific mental 
effort and conscious strategy by users during gamma neurofeedback (unconscious learning) leads to more success in gamma self-regulation learning.

\section{Ethical Considerations}

\section{Compliance with ethical guidelines}

All ethical principles are considered in this article. The participants were informed about the purpose of the research and its implementation stages; they were also assured about the confidentiality of their information; moreover, they were free to leave the study whenever they wished, and if desired, the research results would be available to them.

\section{Funding}

This research did not receive any grant from funding agencies in the public, commercial, or non-profit sectors.

\section{Authors' contributions}

Both authors were equally contributed in preparing this article.

\section{Conflict of interest}

The authors declared no conflict of interest.

\section{References}

Angelakis, E., Stathopoulou, S., Frymiare, J. L., Green, D. L., Lubar, J. F., \& Kounios, J. (2007). EEG neurofeedback: A brief overview and an example of peak alpha frequency training for cognitive enhancement in the elderly. The Clinical Neuropsychologist, 21(1), 110-29. [DOI:10.1080/13854040600744839] [PMID]

Arns, M., Kleinnijenhuis, M., Fallahpour, K., \& Breteler, R. (2008). Golf performance enhancement and real-life neurofeedback training using personalized event-locked EEG profiles. Journal of Neurotherapy, 11(4), 11-8. [DOI:10.1080/10874200802149656]

Başar-Eroglu, C., Strüber, D., Schürmann, M., Stadler, M., \& Başar, E. (1996). Gamma-band responses in the brain: A short review of psychophysiological correlates and functional significance. International Journal of Psychophysiology, 24(1-2), 10112. [DOI:10.1016/S0167-8760(96)00051-7]

Berner, I., Schabus, M., Wienerroither, T., \& Klimesch, W. (2006). The significance of sigma neurofeedback training on sleep spindles and aspects of declarative memory. Applied Psychophysiology and Biofeedback, 31(2), 97-114. [DOI:10.1007/s10484006-9013-7] [PMID] [PMCID]
Birbaumer, N., Ruiz, S., \& Sitaram, R. (2013). Learned regulation of brain metabolism. Trends in Cognitive Sciences, 17(6), 295302. [DOI:10.1016/j.tics.2013.04.009] [PMID]

Bird, B. L., Newton, F. A., Sheer, D. E., \& Ford, M. (1978). Biofeedback training of 40-Hz EEG in humans. Biofeedback and Self-Regulation, 3(1), 1-11. [DOI:10.1007/BF00998559]

Blankertz, B., Sannelli, C., Halder, S., Hammer, E. M., Kübler, A., \& Müller, K.-R., et al. (2010). Neurophysiological predictor of SMR-based BCI performance. NeuroImage, 51(4), 1303-9. [DOI:10.1016/j.neuroimage.2010.03.022] [PMID]

Burde, W., \& Blankertz, B. (2006). Is the locus of control of reinforcement a predictor of brain-computer interface performance? Paper presented at the Proceedings of the $3^{\text {rd }}$ International BrainComputer Interface Workshop and Training Course. https:// graz.pure.elsevier.com/en/publications/proceedingsof-the-3rd-international-brain-computer-interface-wor

Cattell, R. B., Krug, S. E., \& Barton, K. (1973). Technical supplement for the Culture Fair Intelligence Tests, Scales 2 and 3 Champaign, IL: Institute for Personality and Ability Testing.

Daum, I., Rockstroh, B., Birbaumer, N., Elbert, T., Canavan, A., \& Lutzenberger, W. (1993). Behavioural treatment of slow cortical potentials in intractable epilepsy: Neuropsychological predictors of outcome. Journal of Neurology, Neurosurgery $\mathcal{E}$ Psychiatry, 56(1), 94-7. [DOI:10.1136/jnnp.56.1.94] [PMID] [PMCID]

Drechsler, R., Straub, M., Doehnert, M., Heinrich, H., Steinhausen, H. C., \& Brandeis, D. (2007). Controlled evaluation of a neurofeedback training of slow cortical potentials in children with Attention Deficit/Hyperactivity Disorder (ADHD). Behavioral and Brain Functions, 3(1), 35. [DOI:10.1186/1744-90813-35] [PMID] [PMCID]

Egner, T., \& Gruzelier, J. H. (2003). Ecological validity of neurofeedback: Modulation of slow wave EEG enhances musical performance. NeuroReport, 14(9), 1221-4. [DOI:10.1097/00001756-200307010-00006] [PMID]

Egner, T., \& Gruzelier, J. H. (2004). EEG Biofeedback of low beta band components: Frequency-specific effects on variables of attention and event-related brain potentials. Clinical Neurophysiology, 115(1), 131-9. [DOI:10.1016/S1388-2457(03)00353-5]

Elliott, M. A., \& Müller, H. J. (1998). Synchronous Information Presented in 40-HZ Flicker Enhances Visual Feature Binding. Psychological Science, 9(4), 277-83. [DOI:10.1111/14679280.00055]

Enriquez-Geppert, S., Huster, R. J., Scharfenort, R., Mokom, Z., Figge, C., Zimmermann, J., \& Herrmann, C. S. (2013). The morphology of midcingulate cortex predicts frontal-midline theta neurofeedback success. Frontiers in Human Neuroscience, 7, 453. [DOI:10.3389/fnhum.2013.00453] [PMID] [PMCID]

Gevensleben, H., Holl, B., Albrecht, B., Vogel, C., Schlamp, D. \& Kratz, O., et al. (2009). Is neurofeedback an efficacious treatment for ADHD? A randomised controlled clinical trial. Journal of Child Psychology and Psychiatry, 50(7), 780-9. [DOI:10.1111/j.1469-7610.2008.02033.x] [PMID]

Gruzelier, J. (2000). Self regulation of electrocortical activity in schizophrenia and schizotypy: A review. Clinical EEG and Neuroscience, 31(1), 23-9. [DOI:10.1177/155005940003100108] [PMID] 
Halder, S., Varkuti, B., Bogdan, M., Kübler, A., Rosenstiel, W., Sitaram, R., \& Birbaumer, N. (2013). Prediction of braincomputer interface aptitude from individual brain structure. Frontiers in Human Neuroscience, 7, 105. [DOI:10.3389/fnhum.2013.00105] [PMID] [PMCID]

Hammer, E. M., Halder, S., Blankertz, B., Sannelli, C., Dickhaus, T., \& Kleih, S., et al. (2012). Psychological predictors of SMR-BCI performance. Biological Psychology, 89(1), 80-6. [DOI:10.1016/j.biopsycho.2011.09.006] [PMID]

Hammond, D. C. (2000). What do we know about $40 \mathrm{~Hz}$ activity and the tunction it serves? Journal of Neurotherapy: Investigations in Neuromodulation, Neurofeedback and Applied Neuroscience, 4(2), 95-104. [DOI:10.1300/J184v04n02_10]

Hanslmayr, S., Sauseng, P., Doppelmayr, M., Schabus, M., \& Klimesch, W. (2005). Increasing individual upper alpha power by neurofeedback improves cognitive performance in human subjects. Applied Psychophysiology and Biofeedback, 30(1), 1-10. [DOI:10.1007/s10484-005-2169-8] [PMID]

Hommel, B. (1998). Event files: Evidence for automatic integration of stimulus-response episodes. Visual Cognition, 5(1/2), 183-216. [DOI:10.1080/713756773]

Huster, R. J., Mokom, Z. N., Enriquez-Geppert, S., \& Herrmann, C. S. (2013). Brain-computer interfaces for EEG neurofeedback: Peculiarities and solutions. International Journal of Psychophysiology, 91(1), 36-45. [DOI:10.1016/j.ijpsycho.2013.08.011] [PMID]

Keizer, A. W., Verment, R. S., \& Hommel, B. (2010). Enhancing cognitive control through neurofeedback: A role of gammaband activity in managing episodic retrieval. NeuroImage, 49(4), 3404-13. [DOI:10.1016/j.neuroimage.2009.11.023] [PMID]

Keizer, A. W., Verschoor, M., Verment, R. S., \& Hommel, B. (2010). The effect of gamma enhancing neurofeedback on the control of feature bindings and intelligence measures. International Journal of Psychophysiology, 75(1), 25-32. [DOI:10.1016/j. ijpsycho.2009.10.011] [PMID]

Khodakarami, Z., \& Firoozabadi, S. M. P. (2014). [Self-regulation of brain gamma band activity through neurofeedback and its effects on visual feature bindings in healthy female students (Persian)]. Koomesh, 16(1), 36-45. http:/ / koomeshjournal.semums.ac.ir/article-1-2298-fa.html

Kober, S. E., Witte, M., Ninaus, M., Neuper, C., \& Wood, G. (2013). Learning to modulate one's own brain activity: The effect of spontaneous mental strategies. Frontiers in Human Neuroscience, 7, 695. [DOI:10.3389/fnhum.2013.00695] [PMID] [PMCID]

Kübler, A., Blankertz, B., Müller, K., \& Neuper, C. (2011). A model of BCI control. Paper presented at the Proceedings of the $5^{\text {th }}$ International Brain-Computer Interface Conference. https:/ / graz.pure.elsevier.com/en/publications/proceedings-ofthe-5th-international-brain-computer-interface-con

Kübler, A., Neumann, N., Wilhelm, B., Hinterberger, T., \& Birbaumer, N. (2004). Predictability of brain-computer communication. Journal of Psychophysiology, 18(2-3), 121-9. [DOI:10.1027/0269-8803.18.23.121]

Landers, D. M., Petruzzello, S. J., Salazar, W., Crews, D. J., Kubitz, K. A., \& Gannon, T. L.,et al. (1991). The influence of electrocortical biofeedback on performance in pre-elite archers. Medicine \& Science in Sports \& Exercise, 23(1), 123-9. [DOI:10.1249/00005768-199101000-00018]

Leins, U., Goth, G., Hinterberger, T., Klinger, C., Rumpf, N., \& Strehl, U. (2007). Neurofeedback for children with ADHD: A comparison of SCP and theta/beta protocols. Applied Psychophysiology and Biofeedback, 32(2), 73-88. [DOI:10.1007/s10484007-9031-0] [PMID]

Lubar, J., Swartwood, M., Swartwood, J., \& O'Donnell, P. (1995). Evaluation of the effectiveness of EEG neurofeedback training for ADHD in a clinical setting as measured by changes in T.O.V.A. scores, behavioral ratings, and WISCR performance. Biofeedback and Self-Regulation, 20(1), 83-99. [DOI:10.1007/BF01712768] [PMID]

Moore, N. (2000). A review of EEG biofeedback treatment of anxiety disorders. Clinical EEG (Electroencephalography), 31(1) 1-6. [DOI:10.1177/155005940003100105] [PMID]

Nan, W., Rodrigues, J. P., Ma, J., Qu, X., Wan, F., \& Mak, P. I., et al. (2012). Individual alpha neurofeedback training effect on short term memory. International Journal of Psychophysiology, 86(1), 83-7. [DOI:10.1016/j.ijpsycho.2012.07.182] [PMID]

Nan, W., Wan, F., Vai, M. I., \& Da Rosa, A. C. (2015). Resting and initial beta amplitudes predict learning ability in beta/ theta ratio neurofeedback training in healthy young adults. Frontiers in Human Neuroscience, 9, 677. [DOI:10.3389/fnhum.2015.00677] [PMID] [PMCID]

Neumann, N., \& Birbaumer, N. (2003). Predictors of successful self control during brain-computer communication. Journal of Neurology, Neurosurgery \& Psychiatry, 74(8), 1117-21. [DOI:10.1136/jnnp.74.8.1117] [PMID] [PMCID]

Nijboer, F., Birbaumer, N., \& Kübler, A. (2010). The influence of psychological state and motivation on brain-computer interface performance in patients with amyotrophic lateral sclerosis-a longitudinal study. Frontiers in Neuroscience, 4, 55. [DOI:10.3389/fnins.2010.00055] [PMID] [PMCID]

Ninaus, M., Kober, S., Witte, M., Koschutnig, K., Neuper, C., \& Wood, G. (2015). Brain volumetry and self-regulation of brain activity relevant for neurofeedback. Biological Psychology, 110, 126-33. [DOI:10.1016/j.biopsycho.2015.07.009] [PMID]

Raymond, J., Sajid, I., Parkinson, L., \& Gruzelier, J. (2005). Biofeedback and dance performance: A preliminary investigation. Applied Psychophysiology and Biofeedback, 30(1), 6573. [DOI:10.1007/s10484-005-2175-x] [PMID]

Raymond, J., Varney, C., Parkinson, L. A., \& Gruzelier, J. H. (2005). The effects of alpha/theta neurofeedback on personality and mood. Cognitive Brain Research, 23(2-3), 287-92. [DOI:10.1016/j.cogbrainres.2004.10.023] [PMID]

Rubik, B. (2011). Neurofeedback-enhanced gamma brainwaves from the prefrontal cortical region of meditators and nonmeditators and associated subjective experiences. The Journal of Alternative and Complementary Medicine, 17(2), 109-15. [DOI:10.1089/acm.2009.0191] [PMID]

Sams, M. W. (1995). Mathematically derived frequency correlates in cerebral function: Theoretical and clinical implications for neurofeedback treatment. Journal of Neurotherapy, 1(2), 1-14. [DOI:10.1300/J184v01n02_01]

Staufenbiel, S. M., Brouwer, A. M., Keizer, A. W., \& van Wouwe, N. C. (2014). Effect of beta and gamma neurofeedback on 
memory and intelligence in the elderly. Biological Psychology, 95, 74-85. [DOI:10.1016/j.biopsycho.2013.05.020] [PMID]

Tallon-Baudry, C., \& Bertrand, O. (1999). Oscillatory gamma activity in humans and its role in object representation. Trends in Cognitive Sciences, 3(4), 151-62. [DOI:10.1016/S13646613(99)01299-1]

Tallon-Baudry, C., Bertrand, O., Peronnet, F., \& Pernier, J. (1998). Induced $\gamma$-band activity during the delay of a visual shortterm memory task in humans. The Journal of Neuroscience, 18(11), 4244-54. [DOI:10.1523/JNEUROSCI.18-11-04244.1998] [PMID] [PMCID]

Valentin Kvist, A., \& Gustafsson, J. E. (2008). The relation between fluid intelligence and the general factor as a function of cultural background: A test of Cattell's Investment theory. Intelligence, 36(5), 422-36. [DOI:10.1016/j.intell.2007.08.004]

van Boxtel, G. J. M., Denissen, A. J. M., Jäger, M., Vernon, D., Dekker, M. K. J., \& Mihajlović, V., et al. (2012). A novel selfguided approach to alpha activity training. International Journal of Psychophysiology, 83(3), 282-94. [DOI:10.1016/j. ijpsycho.2011.11.004] [PMID]

Vernon, D., Egner, T., Cooper, N., Compton, T., Neilands, C., \& Sheri, A., et al. (2003). The effect of training distinct neurofeedback protocols on aspects of cognitive performance. International Journal of Psychophysiology, 47(1), 75-85. [DOI:10.1016/ S0167-8760(02)00091-0]

Wan, F., Nan, W., Vai, M. I., \& Rosa, A. (2014). Resting alpha activity predicts learning ability in alpha neurofeedback. Frontiers in Human Neuroscience, 8, 500. [DOI:10.3389/fnhum.2014.00500] [PMID] [PMCID]

Watkins, M. W., Lei, P. W., \& Canivez, G. L. (2007). Psychometric intelligence and achievement: A cross-lagged panel analysis. Intelligence, 35(1), 59-68. [DOI:10.1016/j.intell.2006.04.005]

Weber, E., Köberl, A., Frank, S., \& Doppelmayr, M. (2011). Predicting successful learning of SMR neurofeedback in healthy participants: Methodological considerations. Applied Psychophysiology and Biofeedback, 36(1), 37-45. [DOI:10.1007/s10484010-9142-x] [PMID]

Witte, M., Kober, S. E., Ninaus, M., Neuper, C., \& Wood, G. (2013). Control beliefs can predict the ability to up-regulate sensorimotor rhythm during neurofeedback training Frontiers in Human Neuroscience, 7, 478. [DOI:10.3389/fnhum.2013.00478] [PMID] [PMCID] 